\title{
Research on the Thermal Effect of Long Pulse Laser Irradiation BK7 Glass
}

\author{
Yuanyuan Sun ${ }^{1, \mathrm{a}}$, Zheng $\mathrm{Li}^{2, \mathrm{~b}}$, Limin Song $^{3, \mathrm{c}}$, Qiurui Jia ${ }^{4, \mathrm{~d}}$, Shibo Liu $^{5, \mathrm{e}}$ \\ ${ }^{1-4}$ Aviation University of Air Force \\ ${ }^{5}$ Hydrology and Water Resources Bureau \\ Changchun,China \\ ayuanyuansun82@126.com, ${ }^{\mathrm{b}} 1 \mathrm{z} 282332718 @ 126 . c o m,{ }^{\mathrm{c}} 35651633 @$ @q.com, ${ }^{\mathrm{d}}$ jiaqiurui@yahoo.com.cn, ${ }^{\mathrm{e}}$ liushibo_001@163.com
}

\begin{abstract}
The temperature and thermal stress distribution on BK7 glass was numerical simulated by MATLAB according to heat conduction theory. Research has shown that the laser pulse number, spot radius and pulse width can change the extent of the damage threshold for BK7 glass. The results shown the damage threshold decreased with the laser pulse number and radius increasing. The laser power also increased with the pulse width reducing. At the same time, the damage threshold was corresponding reduced.
\end{abstract}

Keywords- BK7 glass, damage threshold, the long pulse laser, irradiation, thermal effect

\section{INTRODUCTION}

Because the optical window of optical system was often irradiated by high energy laser makes the heat accumulation of the optical window internal, thus the optical window caused melt and burst ${ }^{[1-6]}$. The development of high power laser was restricted in the maximum extent, so study the damage characteristic of the optical window is awfully necessary. BK7 glass was an excellent performance of optical materials played a very important role in the optical system and possessed a high transmittance in the ultraviolet and visible. BK7 glass was often used in the laser processing of optical windows, mirrors and prisms. This paper established theory model about BK7 glass irradiated by long pulse Gauss beam and discussed the influence of the damage threshold depends on spot size, pulse number and pulse width by MATLAB.

\section{THEORY MODEL}

Figuer1 shown the circular plate type of target material irradiated by Gauss beam. The thickness of the target material was $h$ and the radius was $r$. I represented center power density of Gauss beam. Materials irradiated by Gaussian beam produced by the space non-uniform temperature field with the emergence of the thermal stress. Through the equation of heat conduction and thermal elastic equation can be comprehensive understanding of heat absorption damage and thermal stress damage.

We selected circular plate BK7 glass as experiment sample $(r=2 \mathrm{~cm}, h=2 \mathrm{~mm})$ and established the cylindrical coordinate system with the origin at the center of the laser incident sample surface. Assumption the sample surface was vertical irradiated by Gauss beam $(z=0)$ and the direction of laser irradiation was consistent with $z$ axis. The initial temperature was $T_{0}$ and the center intensity of the Gauss beam was $P .(R=0.5 \mathrm{~cm}, \tau=1 \mathrm{~ms})$ In order to facilitate the calculation the sample surface and side both were adiabatic surfaces. We concluded the temperature field $T(r, z, t)$ of internal sample according to the heat conduction equation:

$\rho c \frac{\partial T}{\partial t}=\frac{\partial}{\partial r}\left(K \frac{\partial T}{\partial r}\right)+\frac{\partial}{\partial r}\left(K \frac{\partial T}{\partial r}\right)+\frac{\partial}{\partial z}\left(K \frac{\partial T}{\partial z}\right)+A(r, z, t)$

Eq.(1), where $\rho, K, c$ respectively expressed density, thermal conductivity and specific heat capacity of BK7 glass in the general situation. The temperature distribution was expressed by $T$ and time was expressed by $t$. The heat generation rate produced by the internal samples heat conduction was expressed by $A(r, z, t)$.

$$
A(r, z, t)=P \cdot \alpha \cdot \beta \cdot e^{-\beta z}
$$

Eq.(2),where $\mathrm{P}$ was Gaussian beam power density, $\alpha$ and $\beta$ respectively was surface absorption coefficient and volumetric absorption coefficient of BK7 glass sample.

Initial condition:

$$
T(r, z, 0)=T_{0}
$$

Boundary conditions:

$$
\left\{\begin{array}{l}
\left.\frac{\partial T}{\partial r}\right|_{r=r}=0 \\
\left.\frac{\partial T}{\partial z}\right|_{z=h=0}=0
\end{array}\right.
$$

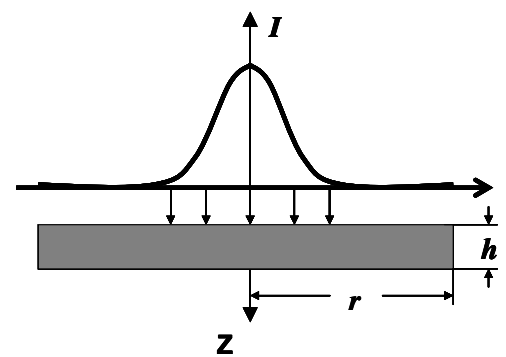

Figure 1. Gaussian beam irradiation circular plate type of target material model

The laser absorption coefficient of BK7 glass was related to glass preparation process, $\mathrm{U}$ Broulik ${ }^{[7]}$ have measured the absorption $\operatorname{coefficient}\left(\alpha=0.46 \sim 0.86 \mathrm{~m}^{-1}\right)$ of BK7 glass samples with $1.06 \mu m$ laser. We selected absorption coefficient $\alpha=0.65 \mathrm{~m}^{-1}$,volumetric absorption 
coefficient ${ }^{[8]} \beta=1.6 \times 10^{5} \mathrm{~m}^{-1}$, concrete parameters of BK7 glass as is shown in table 1.

TABLE.1 BASIC PARAMETERS OF BK7 GLASS

\begin{tabular}{|c|c|}
\hline density & $2.51 \mathrm{~g} / \mathrm{cm}^{3}$ \\
\hline thermal expansion coefficient & $8.6 \times 10^{-6} \mathrm{~K}$ \\
\hline specific heat & $0.71 \mathrm{~J} / \mathrm{g} . \mathrm{K}$ \\
\hline tensile strength & $3 \times 10^{7} \mathrm{~Pa}$ \\
\hline compressive strength & $6.9 \times 10^{8} \mathrm{~Pa}$ \\
\hline young modulus & $8 \times 10^{10} \mathrm{~Pa}$ \\
\hline melting point & $1400^{\circ} \mathrm{C}$ \\
\hline thermal conductivity & $0.0112 \mathrm{w} / \mathrm{cm}^{\circ} \mathrm{C}$ \\
\hline poisson's ratio & 0.208 \\
\hline elasticity modulus & $7.6 \times 10^{-6} \mathrm{~Pa}$ \\
\hline
\end{tabular}

\section{NUMERICAL SIMULATION RESULTS}

\section{A. Temperature distribution}

We got the Gauss temperature distribution of BK7 glass for the laser pulse energy $E$ was $10 \mathrm{~J}$ as shown in Figure2. $(R=0.5 \mathrm{~cm}, \tau=1 \mathrm{~ms})$ Figure 2 shown the highest temperature was in the center of Gaussian spot decreased with radius increasing. The center temperature of Gaussian spot was $230^{\circ} \mathrm{C}$ not reach to the melting point $\left(1400^{\circ} \mathrm{C}\right)$ of BK7 glass ,which not caused melting phenomenon.

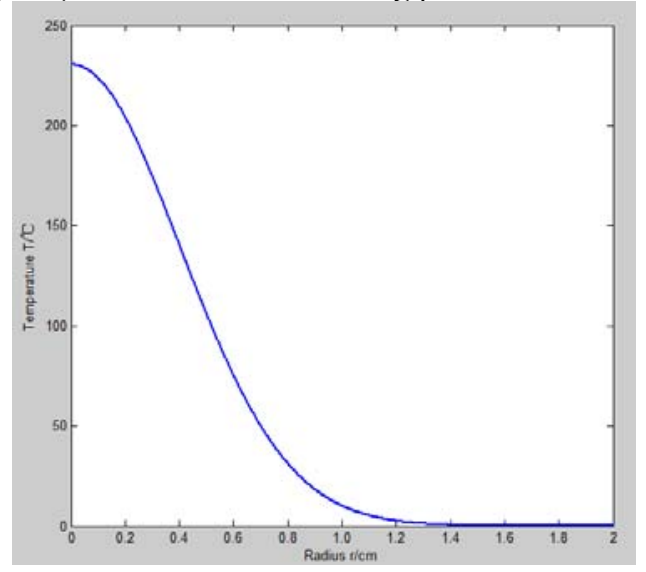

Figure 2. Gauss temperature distribution on BK7 glass

\section{B. Thermal stress distribution}

We also gained the Gaussian thermal stress distribution of BK7 glass through simulations as shown in Figuer3. Figuer $(3,4,5)$ shown the radial and circumferential thermal stress distribution of BK7 glass samples. Because the axial thermal stress component distribution was the same to the radial and circumferential thermal stress component not been discussed here. Compression stress of BK7 glass was controlled by the radial component of thermal stress decreased with radius increasing. When the highest circumferential direction stress was $6.5 \times 10^{7} \mathrm{~Pa}$ not reach the compressive strength $\left(6.9 \times 10^{8} \mathrm{~Pa}\right)$ of the $\mathrm{BK} 7$ glass, so the compression failure phenomenon didn't happen.

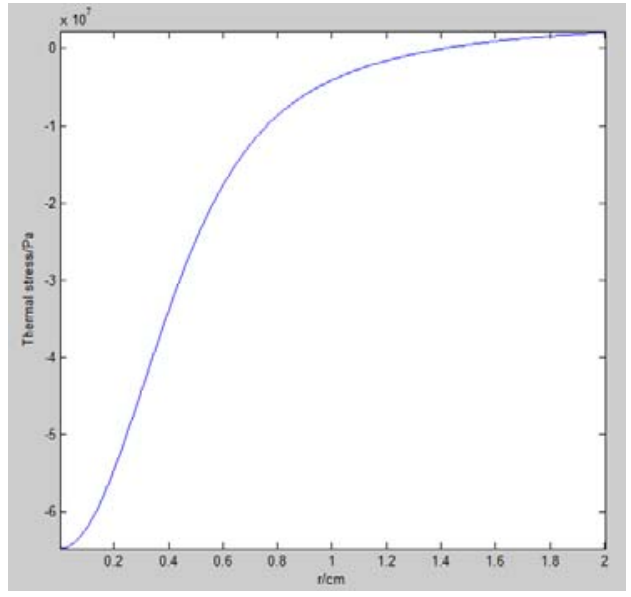

Figure 3. Radial thermal stress distribution on BK7 Glass

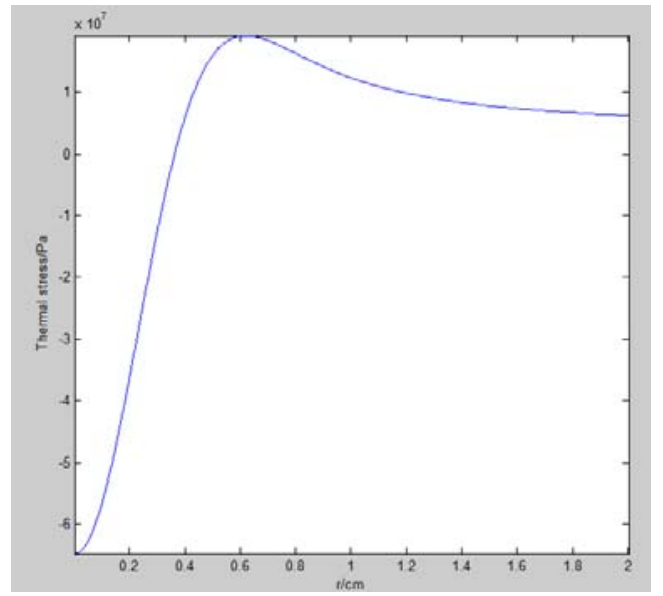

Figure 4. Circumferential thermal stress distribution on BK7 Glass

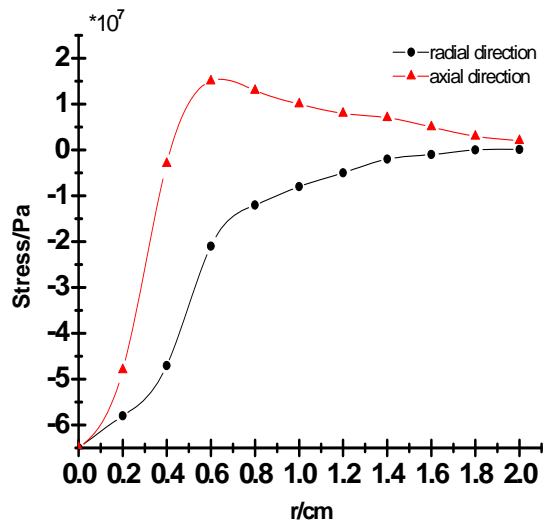

Figure 5. Thermal stress distribution on BK7 Glass

The circumferential thermal stress component was manifested as compression stress of BK7 glass decreased to 0 with radius increasing. The circumferential thermal stress component was manifested as tensile stress in the spot edge region changed from high to low finally closing to a 
minimum value $(0)$ with the increases of the radius of the sample. At this time the highest tensile stress was $1.8 \times 10^{7} \mathrm{~Pa}$ not reach the highest tensile strength $\left(3 \times 10^{7} \mathrm{~Pa}\right)$ of the BK7 glass, so the tension failure phenomenon didn't happen.

\section{Laser pulse number influence on the damage threshold}

We discussed the relationship between the damage threshold and laser pulses number with the spot radius and pulse width unchanged as shown in Figuer6 ( $\tau=1 \mathrm{~ms}$, the spot radius $R=0.5 \mathrm{~cm}$ ). The results shown the damage threshold obviously decreased with radius increasing. After the fifth pulse its downward trend becomes flat.



Figure 6. relationship between the damage threshold and laser pulses number

\section{Radius of Gauss laser influence on the damage threshold}

We got the relationship between the radius of Gauss laser and damage threshold with the pulse width and laser power density unchanged as shown in Figuer7. ( $\tau=1 \mathrm{~ms}, R=0 \sim 1 \mathrm{~cm})$

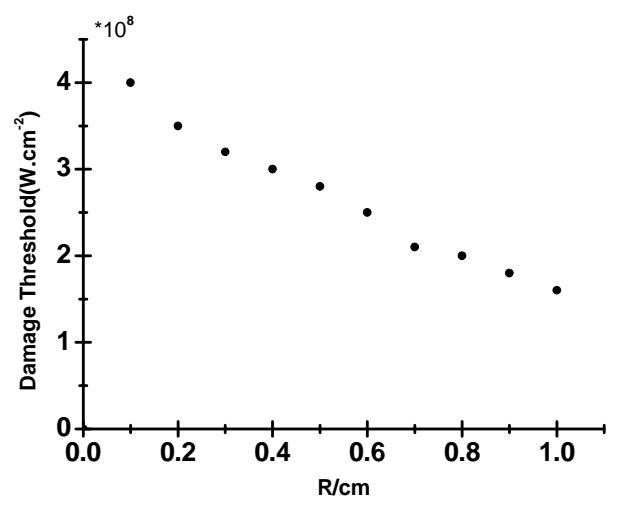

Figure 7. the relationship between the damage threshold and radius of Gauss laser

The results shown damage threshold was changed by spot radius and decreased with radius increasing by researching many radius of sample.

\section{E. Laser pulse width influence on the damage threshold}

The relationship curve between the laser pulse width and damage threshold was gained with the radius of Gauss laser and laser power density unchanged as shown in Figuer8. ( $\tau=0 \sim 1 \mathrm{~ms}, \quad R=0.5 \mathrm{~cm}$ ) The results shown the damage threshold was changed by pulse width which decreased with laser power increasing. Thus, the damage effect was obvious by laser field effect on BK7 glass and the damage threshold was corresponding reduced.

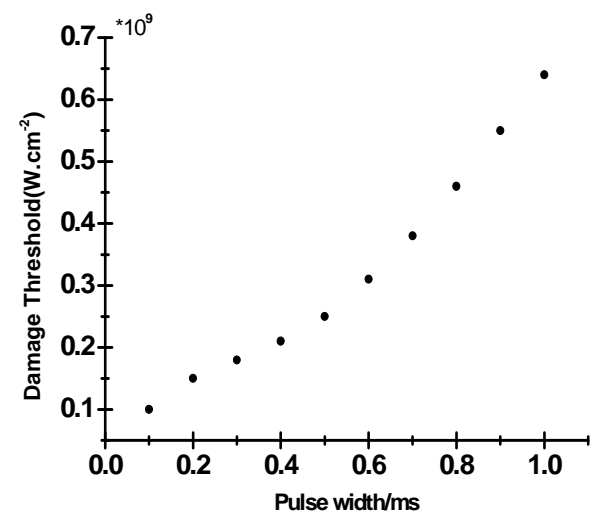

Figure 8. the relationship between the laser pulse width and damage threshold

\section{CONCLUSION}

This paper established the theory model of Gaussian beam irradiation BK7 glass and numerical simulated the temperature and stress field distribution on BK7 glass. The results shown that the thermal stress damage occurred before the melt damage. The circumferential thermal stress mainly manifested as tensile and compression phenomenon which is the major factors in control of BK7 glass damage. This results are as same as other literature ${ }^{[4]}$ reported. Research shown that the pulse number, laser spot radius and laser pulse width of the BK7 glass damage has great influence on damage threshold.

The theoretical model of this paper was also suitable for similar optical element damage problem provided a theoretical foundation for laser processing and laser propulsion field.

\section{REFERENCES}

[1] SUN Chengwei. Laser irradiation effects[M].BEI JING:National Defence Industry Press, 2002

[2] YAN Zongda,WANG Hongli. Thermal sterss[M].BEI JING:China Higher Education Press, 1993

[3] ZHAO Jianjun,SONG Chunrong,NIU Yanxiong. Research on thermal effect of high power laser irradiation optical materials[J].Laser Journal, 2005,26(1):31 34

[4] ZHENG Zhi,JIANG Xiaodong,ZU Xiaotao et al.Research Progress on Properties of Optical Materials Irradiated by Repetition-rate Pulse Laser[J]. Materials Review, 2010,24(12):8〜14 
[5] QIU Rong, WANG Junbo, LI Xiaohong et al.Laser-induced damage on K9 surface under nanotosecond irradiation[J].High Power Laser and Particle Beams. 2011,8 (23): :2101 2105

[6] TAN Yong,CAI Hongxing,ZHANG Xihe et al.Micro-transmittance detection of optic glasses damaged by laser[J].Laser \& Infrared, 2011,12(12):1351 1354
[7] Broulik U.A Comparison between CWand Pulsed Laser Calorimetric Measurement at $1.06 \mu \mathrm{m}$.[A].Guenther A H.SPIE 3244[C].Boulder, Colorado, USA:NIST, 1998:32-41

[8] YANG Shiming,TAO Wenquan.Heat Transfer[M].BEI JING:Higher Education Press,2006 